\title{
Concentración de metales pesados y calidad físico-química del agua de la Ciénaga Grande de Santa Marta
}

\section{Heavy metals concentration and physical-chemical water quality of the Ciénaga Grande de Santa Marta}

\author{
Sonia Esperanza Aguirre ${ }^{1}$; Nelson Virgilio Piraneque ${ }^{2}$; Jean Linero-Cueto ${ }^{3}$
}

\begin{abstract}
1Ing. Agrónoma, M.Sc., Ph.D. Universidad del Magdalena, Ingeniería Ambiental. Santa Marta - Magdalena, Colombia; e-mail: saguirre@unimagdalena.edu.co; (D) https://orcid.org/0000-0002-6975-1940

${ }^{2}$ Ing. Agrónomo, Ph.D. Universidad del Magdalena, Ingeniería Agronómica. Santa Marta - Magdalena, Colombia; e-mail: npiraneque@unimagdalena.edu.co; (D) https://orcid.org/0000-0002-4264-9428

${ }^{3}$ Lic. en Ciencias Físico - Matemáticas, M.Sc., Ph.D. Universidad del Magdalena, Ingeniería Ambiental. Santa Marta - Magdalena, Colombia; e-mail: jlineroc@ unimagdalena.edu.co; (1D) https://orcid.org/0000-0002-2868-4884
\end{abstract}

Cómo citar: Aguirre, S.E.; Piraneque, N.V.; Linero-Cueto, J. 2021. Concentración de metales pesados y calidad físico-química del agua de la Ciénaga Grande de Santa Marta. Rev. U.D.C.A Act. \& Div. Cient. 24(1):e1313. http://doi.org/10.31910/rudca.v24.n1.2021.1313

Artículo de acceso abierto publicado por Revista U.D.C.A Actualidad \& Divulgación Científica, bajo una licencia Creative Commons CC BY-NC 4.0

Publicación oficial de la Universidad de Ciencias Aplicadas y Ambientales U.D.C.A, Institución de Educación Superior Acreditada de Alta Calidad por el Ministerio de Educación Nacional.

Recibido: agosto 16 de $2020 \quad$ Aceptado: diciembre 17 de $2020 \quad$ Editado por: Ingeborg Zenner de Polanía

\section{RESUMEN}

La Ciénaga Grande de Santa Marta (CGSM) es un importante ecosistema para la biodiversidad del planeta. Las actividades antrópicas generan residuos e incrementan los niveles de contaminantes, afectando la calidad del agua, la salud de la población y del ambiente. Con el objetivo de determinar el contenido de metales pesados y calidad del agua de la CGSM, se analizaron muestras de agua, en nueve sitios para las variables temperatura, sólidos disueltos totales (STD), pH, conductividad eléctrica (CE), salinidad, $\mathrm{NO}_{3}{ }^{-}, \mathrm{NH}_{4}{ }^{+}$, $\mathrm{PO}_{4}{ }^{3-}, \mathrm{Ca}^{+2}, \mathrm{Mg}^{+2}, \mathrm{Na}^{+}, \mathrm{SO}_{4}{ }^{2-}, \mathrm{CO}_{3}{ }^{2-}, \mathrm{HCO}_{3}{ }^{-}, \mathrm{Ni}, \mathrm{Cd}, \mathrm{Cr}, \mathrm{Pb}$, Coliformes totales y termotolerantes. La concentración de $\mathrm{Pb}, \mathrm{Cd}, \mathrm{Cr}$ y Ni fue más baja que el valor de referencia para agua marina. La máxima concentración de $\mathrm{Pb}$ y $\mathrm{Cd}$, se presentó en el sitio 6, con 17,76 y $0,48 \mathrm{ppb}$, respectivamente. La salinidad correlacionó con $\mathrm{Ni}$ $(\mathrm{r}=0,98), \mathrm{pH}$ con contenidos de $\mathrm{Ni}$ y $\mathrm{Cr}(\mathrm{r}=0,99$ y 0,43 , respectivamente), $\mathrm{OD}$ se asoció con concentración de $\mathrm{Cd}$ y $\mathrm{Pb}(\mathrm{r}=0,72$ y 0,67 , respectivamente), STD se encuentra asociado con NI y $\mathrm{Cr}$ ( $\mathrm{r}=0,97$ y 0,56 , respectivamente), DBO con concentración de Cd $(r=0,79)$. La concentración de metales pesados encontrados constituye una alerta para el ecosistema, debido a la posible biodisponibilidad ante algunas condiciones ambientales y, a la vez, son reflejo de afectación de variables de calidad del agua del estuario, con transporte de materia orgánica, sedimentos y aportes de nutrientes. Los valores elevados de CT y CF afectan la salud del ecosistema.

Palabras clave: Plomo; Cadmio; Sedimento; Estuarios; Contaminación. (CAB Thesaurus).

\section{ABSTRACT}

The Ciénaga Grande de Santa Marta (CGSM) is an important ecosystem for the planet's biodiversity. Anthropic activities increase levels of pollutants and affect the water quality, the inhabitants' 
health and the environment. In order to determine the CGSM heavy metal content and water quality, samples were collected from nine sites. Temperature, total dissolved solids, hydrogen potential $(\mathrm{pH})$, electrical conductivity, salinity, $\mathrm{NH}_{4}{ }^{+}, \mathrm{PO}_{4}{ }^{3-}, \mathrm{Ca}^{+2}, \mathrm{Mg}^{+2}, \mathrm{Na}^{+}$, $\mathrm{SO}_{4}^{2-},{\mathrm{N}-\mathrm{NO}_{3}}^{2}, \mathrm{CO}_{3}{ }^{2-}, \mathrm{HCO}_{3}, \mathrm{Ni}, \mathrm{Cd}, \mathrm{Cr}, \mathrm{Pb}$, total coliforms and thermotolerant coliforms were determined. The $\mathrm{Pb}, \mathrm{Cd}, \mathrm{Cr}$, and $\mathrm{Ni}$ concentrations, did not exceed the reference value for seawater. The maximum concentration of $\mathrm{Pb}$ and $\mathrm{Cd}$ were observed in point 6 with $17.76 \mathrm{ppb}$ and $0.48 \mathrm{ppb}$, respectively. Salinity correlated with $\mathrm{Ni}(0.98), \mathrm{pH}$ with $\mathrm{Ni}$ and $\mathrm{Cr}$ contents $(\mathrm{r}=0.99$ and 0.43 , respectively), OD was associated with $\mathrm{Cd}$ and $\mathrm{Pb}$ concentration $(\mathrm{r}=0.72$ and 0.67 , respectively), TDS is associated with NI and $\mathrm{Cr}(\mathrm{r}=0.97$ and 0.56 , respectively), BOD with $\mathrm{Cd}$ concentration $(\mathrm{r}=0.79)$. The heavy metals concentrations found, undoubtedly constitute an alert for the ecosystem due to the possible bioaccumulation in some environmental conditions and, it is reflection of the impact on parameters of water quality in the estuary, with the transport of organic matter, sediments, and nutrients inputs. High CT and CF values, affect ecosystem health.

Keywords: Lead; Cadmium; Sediments; Estuary; Pollution.

\section{INTRODUCCIÓN}

La Ciénaga Grande de Santa Marta (CGSM), por sus características hidrológicas, ecológicas y geomorfológicas, es uno de los ecosistemas estuarinos tropicales más grande y productivos del mundo (Yáñez-Arancibia et al. 2014), está protegida por la convención Ramsar y declarada reserva de Biósfera (Martínez et al. 2009). Es un ecosistema dinámico y complejo con fluctuaciones de mareas, que afectan el aporte de sedimentos, alteran la salinidad y las concentraciones de $\mathrm{O}_{2}$ en el agua, condiciones que aumentan la sensibilidad por la intervención antrópica y cambio climático. A su vez, la protección de los ecosistemas acuáticos es esencial para la supervivencia de los mismos. Diversos estudios ponen de manifiesto la necesidad de intervención urgente sobre el complejo lagunar CGSM para consolidar apropiación social del territorio y un trabajo interinstitucional, que unifique esfuerzos para la conservación.

Por otra parte, se ha reportado que la construcción de la carretera Barranquilla-Santa Marta generó muerte de manglares, lo que modificó el ecosistema y alteró su régimen hidrológico, perturbando su funcionalidad. Aunado a esto, la CGSM ha sido sede de conflictos e intereses económicos territoriales de autonomía particular y formas para-estatales que, por violencia, eliminó lo público y fomentó bandas criminales con el monopolio de los recursos naturales (INDEPAZ, 2012). Sus pobladores, pueblos palafitos, subsisten de la pesca; durante el recorrido, se observó la ampliación de zonas ganaderas hacia el complejo lagunar afectando a sus pobladores y el flujo natural de ríos y caños, que son desviados para la agricultura (banano, palma y arroz) (Sarmiento, 2016); dichos cambios afectan el régimen hídrico y alteran la calidad del agua. Campos (1990) argumentó que la presencia de metales pesados en la CGSM afecta cadenas tróficas e indicó la necesidad de monitorear el fenómeno.
El cambio del uso del suelo y la deforestación alrededor de la CGSM, redujo filtros naturales, lo que incrementó la contaminación difusa, escorrentía y erosión generada por la agricultura aledaña al complejo y que es considera una de las fuentes de nitrógeno inorgánico disuelto $\left(\mathrm{NO}_{2}{ }^{-}, \mathrm{NO}_{3}{ }^{-}, \mathrm{NH}_{4}{ }^{+}\right)$, ortofosfatos $\left(\mathrm{PO}_{4}{ }^{3}\right)$ y solidos totales, que llegan por lixiviación al complejo lagunar. Asimismo, los ríos de la Sierra Nevada de Santa Marta y del río Magdalena transportan carga de contaminantes, entre los que se encuentran metales pesados (Campos, 1990). Como consecuencia, la CGSM presenta continuas intervenciones y notables variaciones de la cantidad de oxígeno, salinidad y calidad del agua, oscilaciones que se incrementan en determinadas épocas del año y aumentan el conflicto ambiental (Vivas-Aguas et al. 2013; Rodríguez-Rodríguez et al. 2016; Pérez Ortega et al. 2018).

Regularmente, la presencia de metales en ecosistemas acuáticos se atribuye a geología, características fisicoquímicas del medio y actividades antrópicas. Metales, como $\mathrm{Pb}, \mathrm{Cd}, \mathrm{Cr}, \mathrm{Cu}, \mathrm{Zn}$ y Ni, se acumulan en el suelo, pero por diversos mecanismos, se reducen y pueden ser absorbidos por vegetales o transportados al agua, donde se adhieren a sedimentos a la materia orgánica o por fluctuaciones ambientales, como alteraciones de $\mathrm{pH}$, potencial redox, salinidad, carbonatos, temperatura entre otros, que se pueden liberar y ser biodisponibles. La toxicidad del metal depende de la concentración, la movilidad y su reacción con otros factores, a tener en cuenta en el entorno (Caporale \& Violante, 2016).

La concentración total de metales pesados contenidos en lodos es un posible indicador de contaminación, pero no todas las especies de los mismos, muestran impactos similares, ya que presentan variación en su movilidad o biodisponibilidad, por lo cual, es necesario considerar la fracción retenida en el sedimento y la fracción disuelta en el agua. La retención de metales por material particulado es un proceso significativo para regular la concentración y la distribución de los mismos en ambientes marinos y la formación de complejos órgano-metálicos, es un elemento que regula la biodisponibilidad y toxicidad en ecosistemas acuáticos (Fernández et al. 2009).

El proceso de sedimentación elimina metales del agua y reduce toxicidad, operando el sedimento del fondo como sumidero o trampa; la fracción mineral puede ser transportada en suspensión o con sedimentos, procesos liderados por la dinámica de la floculación y la sedimentación de las partículas (Marcovecchio et al. 2010). Se deduce que la concentración de metales disueltos es comparativamente menor, debido a las transformaciones biogeoquímicas, que regulan su distribución. Asimismo, la fracción disuelta del metal, se puede trasladar por flujo de agua y dispersión (Marcovecchio \& Freije, 2013); sin embargo, los metales solubles en el agua, no siempre estarán biodisponibles, se pueden ligar a la materia orgánica y formar otros complejos, lo que dificulta su determinación.

La concentración de metales en el agua retenida en los espacios de las partículas sólidas de zonas intermareales estuarinas desnudas, aumenta hacia la alcalinidad y la actividad bacteriana (reducción de azufre), incrementando la formación de sulfuros metálicos. 
La oxidación de estos compuestos promueve la disponibilidad del metal y las raíces de la planta los absorbe y cuando la planta muere, se mueve hacia las capas inferiores de los sedimentos (Caetano et al. 2007). Precisamente, Langston et al. (2010) afirmaron que, en algunos estuarios, la concentración de metales en partículas suspendidas no difiere en sedimentos superficiales que mantienen el ciclo del sistema.

La calidad del agua es un indicador clave de sistemas acuáticos, por lo que se planteó caracterizar la calidad del recurso por medio de la concentración de $\mathrm{Ni}, \mathrm{Cd}, \mathrm{Cr}, \mathrm{Pb}$ (ppb), sólidos totales disueltos (STD), $\mathrm{NO}_{3}{ }^{-}, \mathrm{NH}_{4}{ }^{+}, \mathrm{PO}_{4}{ }^{3-} \mu \mathrm{g} \mathrm{L}^{-1}, \mathrm{Ca}^{+2}, \mathrm{Mg}^{+2}, \mathrm{Na}^{+}, \mathrm{SO}_{4}{ }^{2-}, \mathrm{N}-\mathrm{NO}_{3}{ }^{-}, \mathrm{CO}_{3}{ }^{2-}$, $\mathrm{HCO}_{3}{ }^{-}\left(\mathrm{mg} \mathrm{L}^{-1}\right)$, potencial hidrógeno $(\mathrm{pH})$, conductividad eléctrica $\left(\mu \mathrm{S} \mathrm{cm}^{-1}\right)$, salinidad $(\%)$ y temperatura $(\mathrm{T})$ en 9 estaciones del sistema lagunar CGSM, dichas variables, se compararon con los valores establecidos en las normas (Presidencia de la república de Colombia, 2015: Decreto 1076 de 2015) y se analizó su posible afectación.

\section{MATERIALES Y MÉTODOS}

Localización: El trabajo, se desarrolló al Norte de Colombia, complejo lagunar Ciénaga Grande de Santa Marta (CGSM) Santuario de flora y fauna y reserva de biosfera internacional ubicada a 10눙'-

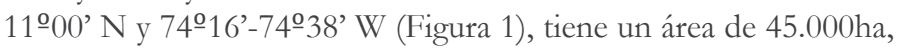
con profundidad media de $150 \mathrm{~cm}$, clima semiárido tropical, con 6 meses secos durante el año y déficit hídrico anual de $1031 \mathrm{~mm}$, dado que la evapotranspiración ( $1431 \mathrm{~mm} /$ año) excede, significativamente, la precipitación (400mm/año), con elevada fluctuación entre años y épocas (seca y de lluvia) (MINAMBIENTE, 2018).

Muestreo: En cada una de las 9 estaciones (Tabla 1), 7 adyacentes a la desembocadura de los ríos y afluentes que drenan sus aguas a la CGSM (Figura 1), se recolectaron cuatro sub-muestras por triplicado, para un total de 81 muestras, en sentido contrario a la corriente en recipiente plástico de 500mL (APHA-AWWA-WEF, 2012), a profundidad de $30 \mathrm{~cm}$. Los sitios fueron georreferenciados (GPS) y las muestras refrigeradas para ser trasladadas al laboratorio del CIAT, para su análisis físico-químico y al laboratorio de aguas de la Universidad del Magdalena, para análisis microbiológico.

Las variables analizadas fueron: conductividad eléctrica - $\mathrm{CE}\left(\mu \mathrm{S} \mathrm{cm}{ }^{-1}\right)$, Temperatura $\left({ }^{\circ} \mathrm{C}\right)$, alcalinidad, $\mathrm{pH}$, dureza, salinidad $(\%)$, coliformes totales CT y coliformes termotolerantes - CF (NMP/100mL), $\mathrm{NO}_{3}{ }^{-}, \mathrm{NH}_{4}{ }^{+}, \mathrm{PO}_{4}{ }^{3-}\left(\mu \mathrm{g} \mathrm{L}^{-1}\right), \mathrm{Ca}^{+2}, \mathrm{Mg}^{+2}, \mathrm{Na}^{+}, \mathrm{SO}_{4}{ }^{2-}, \mathrm{N}-\mathrm{NO}_{3}, \mathrm{CO}_{3}{ }^{2-}$, $\mathrm{HCO}_{3}{ }^{-}\left(\mathrm{mg} \mathrm{L}^{-1}\right), \mathrm{Ni}, \mathrm{Cd}, \mathrm{Cr}, \mathrm{Pb}$ (ppb). Los procedimientos analíticos utilizados, se observan en la tabla 2 y se realizaron con el método estándar para la evaluación de agua y del agua servida, propuesto por APHA-AWWA-WEF (2012). La determinación de oxígeno disuelto y coliformes totales y fecales, se realizó a partir del método de fermentación en tubos múltiples (SM $9221 \mathrm{~B}$ ), 48 horas y $36^{\circ} \mathrm{C}$; se incubó para CT, a $44^{\circ} \mathrm{C}$, para los $\mathrm{CF}$.

Análisis de información: Con los registros obtenidos de las estaciones de muestreo, se construyó la matriz de datos. Para determinar diferencias entre las variables, los datos fueron sometidos a análisis de varianza (ANOVA) y prueba post hoc de comparación de medias de Tukey $(p<0,05)$. Para determinar la asociación entre las variables, se empleó el coeficiente de correlación de Pearson. Se empleó análisis de componentes principales (ACP), a fin de reducir la magnitud de la información. Los datos, se procesaron con el programa estadístico R versión 3.5.0 (R Development Core Team, 2018) e Infostat (2015), en su versión estudiantil.
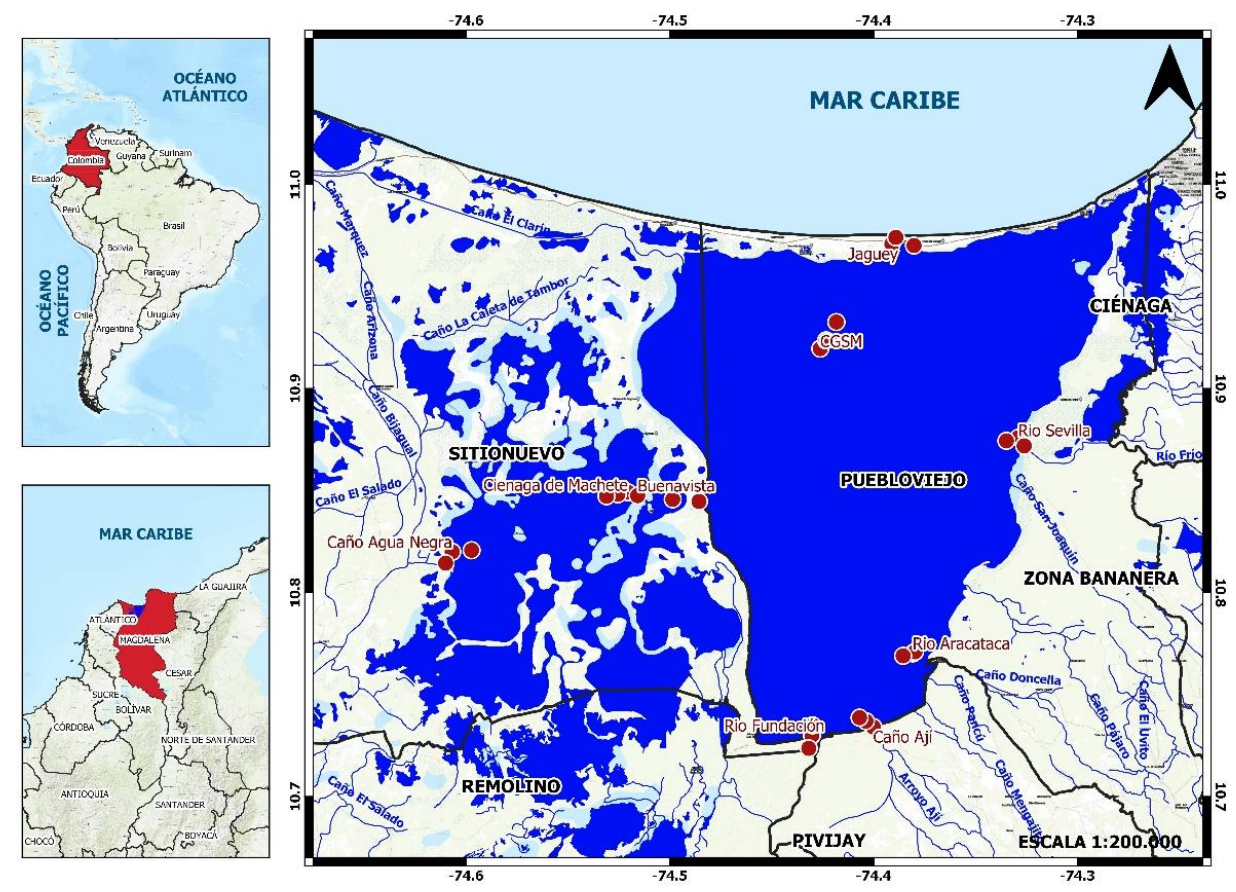

Figura 1. Localización de sitios de muestreo para determinar el contenido de metales pesados y calidad físico-química del agua de la Ciénaga Grande de Santa Marta. 
Tabla 1. Localización de las diferentes estaciones de muestreo empleadas, para determinar la concentración de metales pesados y calidad físico-química del agua de la Ciénaga Grande de Santa Marta.

\begin{tabular}{|c|c|c|c|c|c|}
\hline ESTACIÓN & SECTOR & CARACTERÍSTICAS & PUNTO & LATITUD (N) & LONGITUD (O) \\
\hline \multirow{3}{*}{ E1 } & \multirow{3}{*}{ Río Sevilla } & \multirow{3}{*}{$\begin{array}{l}\text { Influencia del río Vertiente occidental de } \\
\text { la Sierra Nevada de Santa Marta }\end{array}$} & $1 \mathrm{~A}$ & $10^{\circ} 52^{\prime} 33,0^{\prime \prime}$ & $74^{\circ} 19^{\prime} 44,6^{\prime \prime}$ \\
\hline & & & $1 \mathrm{~B}$ & $10^{\circ} 52^{\prime} 18,8^{\prime \prime}$ & $74^{\circ} 19^{\prime} 34,1^{\prime \prime}$ \\
\hline & & & $1 \mathrm{C}$ & $10^{\circ} 52^{\prime} 27,7^{\prime \prime}$ & $74^{\circ} 20^{\prime} 05,5^{\prime \prime}$ \\
\hline \multirow{3}{*}{$\mathrm{E} 2$} & \multirow{3}{*}{ Río Aracataca } & \multirow{3}{*}{$\begin{array}{l}\text { Influencia del río Vertiente occidental de } \\
\text { la Sierra Nevada de Santa Marta }\end{array}$} & $2 \mathrm{~A}$ & $10^{\circ} 46^{\prime} 12,7^{\prime \prime}$ & $74^{\circ} 22^{\prime} 50,8^{\prime \prime}$ \\
\hline & & & $2 \mathrm{~B}$ & $10^{\circ} 46^{\prime} 15,5^{\prime \prime}$ & $74^{\circ} 22^{\prime} 46,9^{\prime \prime}$ \\
\hline & & & $2 \mathrm{C}$ & $10^{\circ} 46^{\prime} 08,3^{\prime \prime}$ & $74^{\circ} 23^{\prime} 08,0^{\prime \prime}$ \\
\hline \multirow{3}{*}{ E3 } & \multirow{3}{*}{ Caño Ají } & \multirow{3}{*}{ Sistema lacustre del complejo lagunar } & $3 \mathrm{~A}$ & $10^{\circ} 44^{\prime} 02,6^{\prime \prime}$ & $74^{\circ} 24^{\prime} 00,6^{\prime \prime}$ \\
\hline & & & $3 \mathrm{~B}$ & $10^{\circ} 44^{\prime} 13,0^{\prime \prime}$ & $74^{\circ} 24^{\prime} 13,7^{\prime \prime}$ \\
\hline & & & $3 \mathrm{C}$ & $10^{\circ} 44^{\prime} 19,2^{\prime \prime}$ & $74^{\circ} 24^{\prime} 24,9^{\prime \prime}$ \\
\hline \multirow{3}{*}{$\mathrm{E} 4$} & \multirow{3}{*}{ Río Fundación } & \multirow{3}{*}{$\begin{array}{l}\text { Influencia del río Vertiente occidental de } \\
\text { la Sierra Nevada de Santa Marta }\end{array}$} & $4 \mathrm{~A}$ & $10^{\circ} 43^{\prime} 47,9^{\prime \prime}$ & $74^{\circ} 25^{\prime} 49,0^{\prime \prime}$ \\
\hline & & & $4 \mathrm{~B}$ & $10^{\circ} 43^{\prime} 25,0^{\prime \prime}$ & $74^{\circ} 25^{\prime} 54,6^{\prime \prime}$ \\
\hline & & & $4 \mathrm{C}$ & $10^{\circ} 44^{\prime} 05,7^{\prime \prime}$ & $74^{\circ} 25^{\prime} 47,9^{\prime \prime}$ \\
\hline \multirow{3}{*}{ E5 } & \multirow{3}{*}{$\begin{array}{l}\text { Ciénaga de Ma- } \\
\text { chete }\end{array}$} & \multirow{3}{*}{ Sistema lacustre del complejo lagunar } & $5 \mathrm{~A}$ & $10^{\circ} 50^{\prime} 56,8^{\prime \prime}$ & $74^{\circ} 31^{\prime} 14,1^{\prime \prime}$ \\
\hline & & & $5 B$ & $10^{\circ} 50^{\prime} 53,3^{\prime \prime}$ & $74^{\circ} 31^{\prime} 31,8^{\prime \prime}$ \\
\hline & & & $5 \mathrm{C}$ & $10^{\circ} 50^{\prime} 49,9^{\prime \prime}$ & $74^{\circ} 31^{\prime} 51,8^{\prime \prime}$ \\
\hline \multirow{3}{*}{ E6 } & \multirow{3}{*}{$\begin{array}{l}\text { Caño Agua } \\
\text { Negra }\end{array}$} & \multirow{3}{*}{ Sistema lacustre del complejo lagunar } & $6 \mathrm{~A}$ & $10^{\circ} 49^{\prime} 11,8^{\prime \prime}$ & $74^{\circ} 36^{\prime} 24,4^{\prime \prime}$ \\
\hline & & & $6 \mathrm{~B}$ & $10^{\circ} 48^{\prime} 51,8^{\prime \prime}$ & $74^{\circ} 36^{\prime} 36,0^{\prime \prime}$ \\
\hline & & & $6 \mathrm{C}$ & $10^{\circ} 49^{\prime} 14,6^{\prime \prime}$ & $74^{\circ} 35^{\prime} 50,1^{\prime \prime}$ \\
\hline \multirow{3}{*}{ E7 } & \multirow{3}{*}{ Buenavista } & \multirow{3}{*}{ Sistema lacustre del complejo lagunar } & $7 \mathrm{~A}$ & $10^{\circ} 50^{\prime} 51,0^{\prime \prime}$ & $74^{\circ} 30^{\prime} 56,8^{\prime \prime}$ \\
\hline & & & $7 \mathrm{~B}$ & $10^{\circ} 50^{\prime} 44,4^{\prime \prime}$ & $74^{\circ} 29^{\prime} 54,9^{\prime \prime}$ \\
\hline & & & $7 \mathrm{C}$ & $10^{\circ} 50^{\prime} 40,7^{\prime \prime}$ & $74^{\circ} 29^{\prime} 08,5^{\prime \prime}$ \\
\hline \multirow{3}{*}{ E8 } & \multirow{3}{*}{$\begin{array}{l}\text { Ciénaga Grande } \\
\text { de Santa Marta } \\
\text { CGSM }\end{array}$} & \multirow{3}{*}{ Cuerpo de agua principal de la CGSM } & $8 \mathrm{~A}$ & $10^{\circ} 55^{\prime} 10,4^{\prime \prime}$ & $74^{\circ} 25^{\prime} 34,3^{\prime \prime}$ \\
\hline & & & $8 \mathrm{~B}$ & $10^{\circ} 55^{\prime} 25,0^{\prime \prime}$ & $74^{\circ} 25^{\prime} 24,0^{\prime \prime}$ \\
\hline & & & $8 \mathrm{C}$ & $10^{\circ} 55^{\prime} 57,3^{\prime \prime}$ & $74^{\circ} 25^{\prime} 05,8^{\prime \prime}$ \\
\hline \multirow{3}{*}{ E9 } & \multirow{3}{*}{ Jagüey } & \multirow{3}{*}{ Sistema lacustre del complejo lagunar } & $9 \mathrm{~A}$ & $10^{\circ} 58^{\prime} 15,1^{\prime \prime}$ & $74^{\circ} 23^{\prime} 27,9^{\prime \prime}$ \\
\hline & & & $9 \mathrm{~B}$ & $10^{\circ} 58^{\prime} 26,7^{\prime \prime}$ & $74^{\circ} 23^{\prime} 20,7^{\prime \prime}$ \\
\hline & & & $9 \mathrm{C}$ & $10^{\circ} 58^{\prime} 12,3^{\prime \prime}$ & $74^{\circ} 22^{\prime} 48,7^{\prime \prime}$ \\
\hline
\end{tabular}

Tabla 2. Procedimientos analíticos y equipos empleados para la caracterización del agua de la CGSM.

\begin{tabular}{|c|c|c|c|}
\hline Parámetro & Técnica Analítica & Equipo & Margen de error \\
\hline $\mathrm{pH}$ & Potenciometría & pH-metro digital Hanna HI2020 & $1 \%$ \\
\hline Conductividad & Electrométrico & Conductímetro Hanna HI2030 & $1 \%$ \\
\hline Nitratos & Electrodo de ion selectivo & $\begin{array}{l}\text { Medidor de iones Ion Meter } 692 \text { con Electrodo Se- } \\
\text { lectivo de Nitrato }\end{array}$ & $5 \%$ \\
\hline Sulfatos & Espectrofotométrico & $\begin{array}{l}\text { Espectrofotómetro ultravioleta-visible UV-VIS a } \\
\text { 420nm }\end{array}$ & $5 \%$ \\
\hline $\begin{array}{l}\mathrm{Ca}, \mathrm{Na}, \mathrm{Mg}, \mathrm{K} . \\
\mathrm{mg} / \mathrm{L}\end{array}$ & Espectrofotometría de absorción atómica & Espectrofotómetro Unicam 969 Solar & $5 \%$ \\
\hline $\begin{array}{l}\mathrm{Pb}, \mathrm{Cd}, \mathrm{Ni}, \mathrm{Cr} . \\
\mathrm{ppb}\end{array}$ & $\begin{array}{l}\text { Espectrometría de emisión óptica con plasma } \\
\text { acoplado inductivamente -digestión en ácido (ICP) }\end{array}$ & ICP-OES Thermo Scientific ${ }^{\mathrm{TM}}$ iCAPTM 7200 DUO & $5 \%$ \\
\hline $\begin{array}{l}\text { Dureza total } \\
\mathrm{mg} / \mathrm{L} \mathrm{CaCO}_{3}\end{array}$ & Volumetría & Titulación con EDTA, SM 2340 -C & $5 \%$ \\
\hline $\begin{array}{l}\text { Alcalinidad total } \\
\mathrm{mg} / \mathrm{L} \mathrm{CaCO}_{3}\end{array}$ & Método Volumétrico & $\mathrm{HCl}, \mathrm{SM} 2320-\mathrm{B}$ & $5 \%$ \\
\hline Turbiedad & Turbidimetría & Turbidímetro digital HANNA hi 93703 - 0-1 FTU & $5 \%$ \\
\hline Color & Colorimetría & Espectrofotómetro & $5 \%$ \\
\hline DQO & Reflujo cerrado & Espectrofotométrico SM 5220 D & $5 \%$ \\
\hline
\end{tabular}


Continuación Tabla 2

\begin{tabular}{|l|l|l|c|}
\hline \multicolumn{1}{|c|}{ Parámetro } & \multicolumn{1}{c|}{ Técnica Analítica } & \multicolumn{1}{c|}{ Equipo } & Margen de error \\
\hline $\begin{array}{l}\text { Coliformes } \\
\text { totales }(\mathrm{CT}) \\
\text { y termotol- } \\
\text { erantes }(\mathrm{CF}) \\
(\mathrm{NMP} / 100 \mathrm{Ml})\end{array}$ & Fermentación en tubos múltiples & (SM 9221, APHA et al. 2005) & \\
\hline Temperatura & & Termométrico SM 2550B & $5 \%$ \\
\hline
\end{tabular}

$\mathrm{SM}=$ Standard Methods

\section{RESULTADOS Y DISCUSIÓN}

Los valores de las variables estudiadas, se registran en la tabla 3. El pH exhibió características propias de agua de mar con valores aceptables para la flora y fauna de aguas cálidas marinas y estuarinas (Hernández-Ayón et al. 2003), el cual, varía entre 5 y 9; sin embargo, no mostró diferencias significativas. El oxígeno disuelto (OD), varió entre 2,78 y $9,7 \mathrm{mg} \mathrm{L}^{-1}$, siendo los valores máximos encontrados en los sitios 7 y 5 (Buenavista y Ciénaga de Machete, respectivamente), lo que puede estar relacionado con intercambios con la atmósfera y a la respiración de organismos fotosintéticos, de los que se observó presencia en el agua. Los mayores valores de Coliformes Totales (CT), se encontraron en las estaciones 7 y 6, con valores de 47683 y $36716 \mathrm{NMP} / 100 \mathrm{~mL}$, respectivamente, mientras que los valores más bajos, se obtuvieron en la estación 1, con 11703 NMP/100mL.

Tabla 3. Características físicas, químicas y biológicas y contenido de metales pesados del agua de la CGSM.

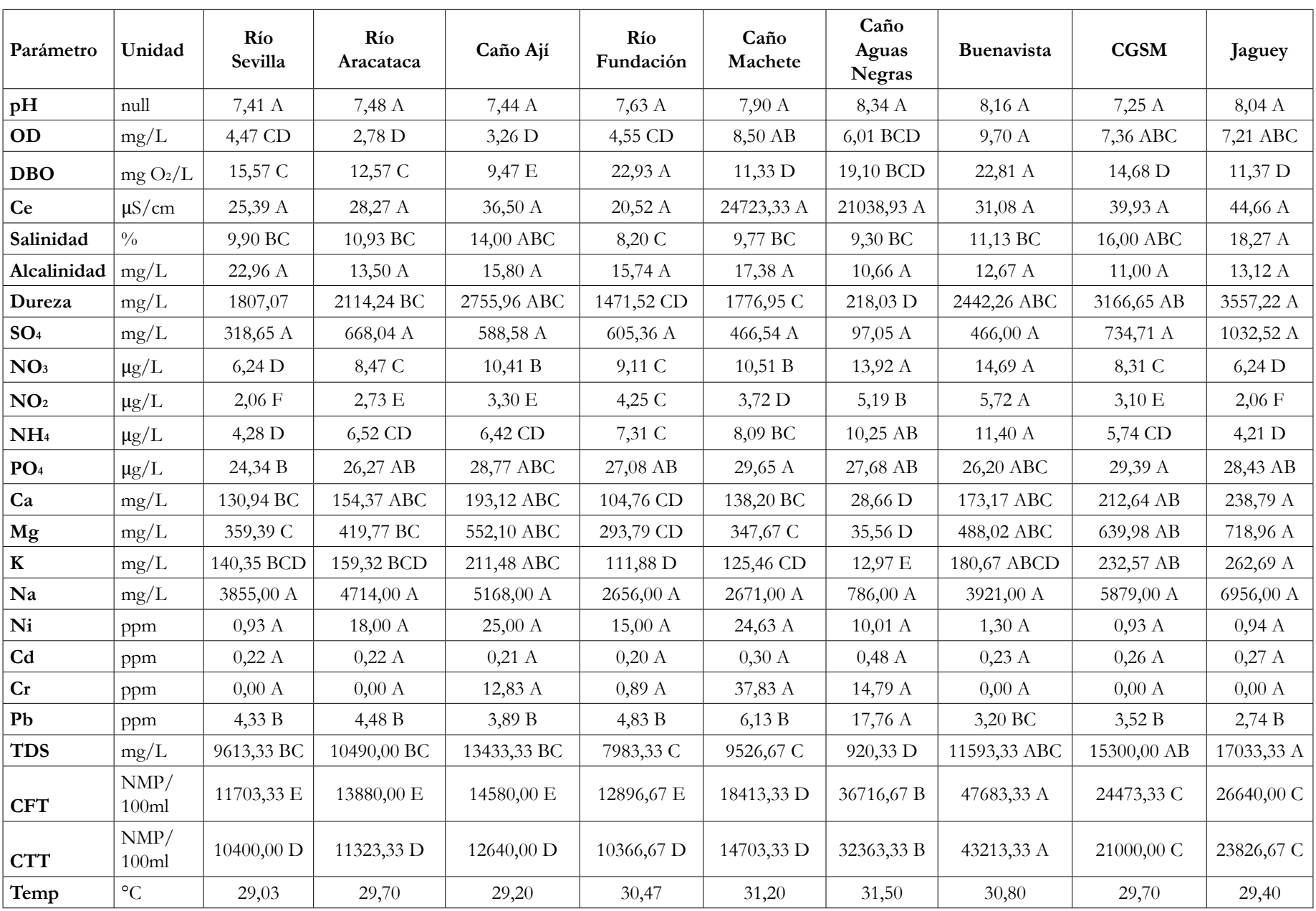

Medias con una letra común no son significativamente diferentes $(\mathrm{p}>0,05)$. 
La CE no presentó diferencias entre sitios, se puede deducir que existe arrastre de elementos disueltos, ya que cambia con la cercanía de las desembocaduras de ríos y caños; esta variable se relaciona con la concentración de SDT y el porcentaje de salinidad, por lo tanto, la aportación de agua dulce participa en el estuario, generando gradientes salinos y estratificación, con transporte de sedimentos, materia orgánica y nutrientes (Marcovecchio \& Freije, 2013), que modifican las características del sistema. El porcentaje de salinidad más alto, se encontró en la estación Jagüey, con 18\%, seguido del 8 , con $16 \%$. De igual manera, estas estaciones presentaron la mayor dureza, con 3557 y $3166 \mathrm{mg} \mathrm{L}^{-1}$, respectivamente, lo que coincide con la concentración de $\mathrm{Na}$ (6956 $\left.\mathrm{mg} \mathrm{L}^{-1}\right)$, en la estación Jagüey y $5879 \mathrm{mg} \mathrm{L}^{-1}$, para la estación CGSM. Estos valores indican que en el estuario predominan condiciones similares a las marinas.

El análisis de correlación expuso relación directa entre contenidos de metales y las demás variables. Ni mostró relaciones significativas con $\mathrm{pH}$, salinidad, CE, TDS, $\mathrm{NO}_{3}, \mathrm{NO}_{2}, \mathrm{NH}_{4}{ }^{+}, \mathrm{Ca}^{+2}, \mathrm{Mg}^{+2}, \mathrm{~K}^{+}, \mathrm{Na}^{+}$ y dureza $(\mathrm{r}=0,99,0,98,0,88,0,97,0,98,0,99,0,74,0,87,0,91,0,85$, 0,88 , y 0,91 , respectivamente). Por su parte, $\mathrm{Cd}$ correlacionó positiva y significativamente con $\mathrm{OD}, \mathrm{DBO}, \mathrm{Ni}$ y $\mathrm{Pb}(\mathrm{r}=0,72,0,79,0,97$ y 0,79 , respectivamente). $\mathrm{Cr}$ correlacionó con $\mathrm{CF}, \mathrm{NO}_{2}^{-}, \mathrm{HCO}_{3}^{-}$y $\operatorname{ALC}(\mathrm{r}=0,72,0,72,0,88$ y 0,83 , respectivamente) y plomo relacionó significativamente con $\mathrm{OD}$ y $\mathrm{Cd}$ ( $\mathrm{r}=0,67$ y 0,63 , respectivamente).

El ACP estableció cuatro componentes que dan cuenta del $82 \%$ de la variabilidad. Con relación al CP1 (SALES), que explica el 42\% de la varianza, se puede destacar que las variables salinidad, CE, TDS, $\mathrm{Ca}^{+2}, \mathrm{Mg}^{+2}, \mathrm{~K}^{+}, \mathrm{Na}^{+}, \mathrm{SO}_{4}^{2-}$ y dureza están relacionados con elevados contenidos de sales. El CP2 (BIOLÓGICO) está explicado en 19\% por las variables $\mathrm{OD}, \mathrm{pH}, \mathrm{CT}$ y $\mathrm{CF}$, estrechamente relacionadas al componente microbiológico; el CP3 (METALES PESADOS), que acumula el $10 \%$ de la varianza, está definido por los contenidos de $\mathrm{Pb}, \mathrm{Ni}$, Cr y Cd y, finalmente, el CP4 (NITRÓGENO Y CARBO$\mathrm{NO}$ ), con el $8 \%$ de la varianza, está determinado por los contenidos de $\mathrm{NH}_{4}{ }^{+}$y $\mathrm{NO}_{3}{ }^{-}$(Figura 2).

Los resultados del estudio evidencian que las variables evaluadas se encuentran en valores normales para agua de condiciones marinas; sin embargo, se debe considerar que el área de análisis es un estuario del plano deltaico del río Magdalena, donde se mezcla agua dulce y salada, que forma el complejo lagunar. El pH no reportó diferencias, osciló entre 7,25 y 8,34, máximo valor presente en el sitio 6, que corresponde al caño Aguas Negras (Tabla 3). Este parámetro afecta la mayoría de los procesos hídricos y, los valores tendientes a la alcalinidad, afectan la vida y favorece la adsorción y precipitación de metales, al unirse con $\mathrm{OH}^{+}$y $\mathrm{HCO}_{3}$, que interactúan entre el agua y sedimentos, siendo susceptibles a ser liberados y transportados fácilmente. Así, un incremento o cambio inesperado en $\mathrm{pH}$, puede interferir la biodisponibilidad de contaminantes (Cuizano et al. 2010), situación que, en esta investigación, se evidencia con la asociación positiva presente, entre esta variable y el contenido de $\mathrm{Ni}$ y $\mathrm{Cr}$.

La concentración de oxígeno disuelto OD es indispensable para la calidad de la vida acuática en lagos y lagunas, condiciona procesos de fermentación, lo que disminuye la calidad del sistema. La variable registró diferencias significativas, siendo las estaciones Buenavista y Jagüey, las que evidenciaron valores superiores a $4 \mathrm{mg} \mathrm{L}^{-1} \mathrm{O}_{2}$. Menor concentración de $\mathrm{OD}$ en agua genera mayor presión, donde valores menores de $2 \mathrm{mg} \mathrm{L}^{-1}$ ocasionan la muerte de peces, por hipoxia o anoxia, evento que se reporta en épocas secas en la CGSM (INVEMAR, 2016a; b). El OD que requieren los organismos, depende de la especie, tamaño y la temperatura del ambiente acuático, entre otros. Si la temperatura del agua aumenta, el oxígeno disminuye. Otro factor que incide es la respiración microbiana por efecto de la degradación de compuestos orgánicos (Pradana et al. 2019).

La fertilización excesiva de los cultivos aledaños a la CGSM (banano y palma de aceite) incrementa la escorrentía de nitratos y fosfatos, lo cual, agudiza el proceso de eutrofización, fenómeno que reduce la concentración de oxígeno. De igual manera, las aguas provenientes de asentamientos humanos incrementan la concentración de bacterias consumidoras de oxígeno y la carga de microorganismos del sistema, situación que se ratifica en relación positiva con CF, donde las máximas concentraciones se mostraron en las estaciones 7 y 6 , con valores de 43000 y $32000 \mathrm{NMP} / 100 \mathrm{~mL}$, respectivamente. Son evidentes los valores elevados de CT y CF, al ser comparadas con los valores aceptables de agua admisible para fines recreativos; el contacto primario o secundario puede ocasionar problemas de salubridad, Decreto 1076 de 2015 (Presidencia de la República de Colombia, 2015). Estos resultados evidencian la baja calidad sanitaria en este complejo lagunar.

Las variables $\mathrm{CE}$, salinidad, concentración de $\mathrm{Na}^{+}, \mathrm{Ca}^{+2}, \mathrm{Mg}^{+2}, \mathrm{SO}_{4}^{2-}$, $\mathrm{PO}_{4}{ }^{3-}$ y dureza están asociadas (Figura 2) y ponen de manifiesto dependencia y proporcionalidad positiva entre ellas. La variable de salinidad presentó alta correlación con concentraciones de bases y TDS, donde la concentración de elementos, como $\mathrm{K}^{+}, \mathrm{Ca}^{2+}, \mathrm{Mg}^{+2}$ y $\mathrm{SO}_{4}{ }^{2-}$, contribuye al aumento de salinidad y, a su vez, sus concentraciones, expusieron diferencias significativas respecto al sitio de muestreo, reflejando la dinámica físico-química del agua de la CGSM, influenciada por la descarga de agua de los ríos Aracataca, Sevilla, Fundación y Magdalena, además del intercambio con el mar adyacente, lo que propicia diferentes gradientes de salinidad del estuario (Tabla 3), fenómeno que argumentó Spetter et al. (2013) y es propio de ecosistemas estuarinos.

El menor porcentaje de salinidad y de concentración de $\mathrm{Na}^{+}$, lo reportó la estación 4 , con $8,2 \%$ y $1.656 \mathrm{mg} \mathrm{L}^{-1} \mathrm{de} \mathrm{Na}^{+}$y la 6 , con 9,3\% y $786 \mathrm{mg} \mathrm{L}^{-1}$ de $\mathrm{Na}^{+}$(Tabla 3). Las estaciones de muestreo CGSM y Jagüey mostraron valores altos de salinidad (16-18\%), que equivalen a 160-180 partes por mil, que corresponden a hipersalinidad, posiblemente, por la cercanía al mar; en esta condición, hay menores tasas de crecimiento vegetal y de diversidad del ecosistema. La elevada concentración de sales disueltas afecta la biota y el equilibrio de la CGSM; no obstante, la salinidad se asocia con variables, como temperatura y precipitación, por lo que oscila constantemente (Kirwan et al. 2009).

En la figura 2, se muestra la dispersión, la distribución y la asociación de las variables y su tendencia. Se observa asociación entre porcentaje de salinidad, fósforo, sulfatos, CE y contenido de $\mathrm{Na}^{+}$, 


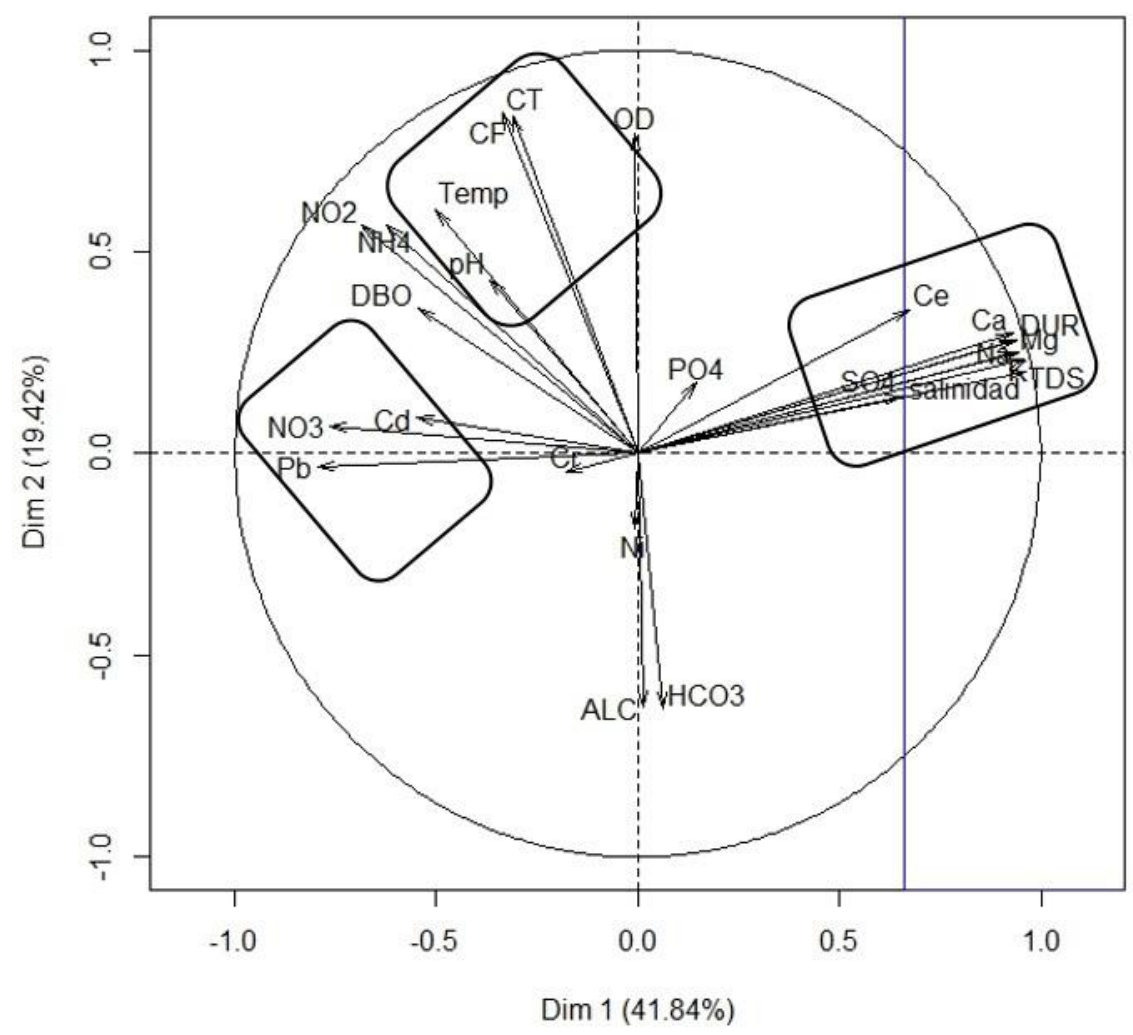

Figura 2. Análisis de componentes principales de las variables medidas, para establecer el contenido de metales pesados y la calidad físico-química del agua de la Ciénaga Grande de Santa Marta.

$\mathrm{Ca}^{+2} \mathrm{y} \mathrm{Mg}^{+2}$. Asimismo, se estableció la relación fuerte entre nitratos, coliformes, $\mathrm{DBO}, \mathrm{pH}$, concentración de $\mathrm{Pb}$ y Cd. Igualmente, se observó la afinidad de las variables, alcalinidad (bicarbonatos) y concentración de $\mathrm{Cr}$ y $\mathrm{Ni}$, lo que puede ser interpretado que, en el momento dichas variables, no representan una contribución importante en la valoración del sistema. La alcalinidad es una medida de la resistencia del agua a reducir $\mathrm{pH}$ cuando se le añade ácido; los resultados no evidenciaron diferencia significativa respecto al sitio de muestreo y presentaron correlación positiva con las concentraciones de $\mathrm{HCO}_{3}{ }^{-}(\mathrm{r}=0,97)$ y $\mathrm{Cr}(\mathrm{r}=0,83)$.

Entre los mayores aportantes de nutrientes en las costas, se encuentra la descarga de afluentes, como los ríos (Spetter et al. 2013). Los nutrientes inorgánicos disueltos $\left(\mathrm{NH}_{4}{ }^{+}, \mathrm{NO}_{3}\right)$, se encontraron en baja concentración (Tabla 3), lo cual, se atribuye, posiblemente, al consumo por parte del fitoplancton (Jaramillo-Londoño \& Aguirre-Ramírez, 2012). Las estaciones CGSM y Jagüey fueron las de mayor concentración de ortofosfatos (Tabla 3), un indicador de la descarga de aguas residuales en la ciénaga. Por otro lado, se observaron diferencias significativas por el intercambio constante de amonio y de fósforo, entre el interior del continente (río arriba) y la concentración del agua y el sedimento (Vivas-Aguas et al. 2013); el incremento de $\mathrm{N}$ y P induce la eutrofización y acelera el crecimiento de algas que agotan oxígeno y por descomposición, cuando mueren, incrementa el problema de eutrofización.
La concentración de $\mathrm{Pb}, \mathrm{Cd}$, Cr y Ni no superó el valor de referencia para agua marina, Decreto, 1076 de 2015 (Presidencia de la República de Colombia, 2015), pero por su toxicidad y biodisponibilidad deben ser monitoreadas y su presencia constituye una alerta para el ecosistema (Beltrame et al. 2009; Reyes et al. 2016). El sitio 6, que corresponde al caño de Aguas Negras, reportó mayor concentración de metales pesados, lo que coincide con el histórico del INVEMAR, que aluden esta situación a la cercanía al río Magdalena, pero de igual forma, no son valores que expresen, por el momento, riesgo ambiental, pero que es conveniente monitorear.

Los máximos niveles de $\mathrm{Cd}$, se reportaron en la estación Caño de aguas negras, con 0,48 ppb, (Tabla 3), indicando que, aunque la concentración es baja, existe la posibilidad de presentar efectos adversos en organismos más sensibles, al estar disueltos en el agua; no obstante, al compararse con niveles de referencia internacionales (Tabla 4), no excede los límites permisibles. Cuando el Cd se oxida al unirse con sulfatos o $\mathrm{Zn}$ existe la posibilidad de generar $\mathrm{Cd}^{+2}$, que es la forma más peligrosa, que aumenta conforme se disminuye el oxígeno disuelto; sin embargo, las características de salinidad del sistema pueden incrementar la tolerancia a la concentración del metal. La estación Caño de aguas negras presentó la alta concentración de plomo $(\mathrm{Pb})$, con $17,76 \mathrm{ppb}$, con diferencias significativas respecto a los demás puntos de muestreo. 
Tabla 4. Concentración de metales pesados en sedimentos de la CGSM.

\begin{tabular}{|c|c|c|c|c|c|c|c|c|c|c|}
\hline \multirow{2}{*}{ Metal } & E1 & E2 & E3 & $\mathbf{E} 4$ & E6 & \multicolumn{4}{|c|}{ Marco de referencia legislación. (ppb) } & \multirow{2}{*}{$\begin{array}{r}\text { Campos } \\
1990\end{array}$} \\
\hline & \multicolumn{5}{|c|}{ ppb } & EPA & CANADA & HOLANDA & CONAMA & \\
\hline $\mathrm{Cr}$ & 4,98 & 4,89 & 5,04 & 3,95 & 8,3 & 2 & 26 & 100 & 500 & ---- \\
\hline $\mathrm{Ni}$ & 6,2 & 7,31 & 16,3 & 4,23 & 15,3 & - & 16 & 35 & 25 & ----- \\
\hline $\mathrm{Cd}$ & 0,02 & 1,04 & 0,5 & 1,07 & $<\mathrm{LD}$ & 2 & - & & 10 & 2,04 \\
\hline $\mathrm{Pb}$ & $>\mathrm{LD}$ & 6,12 & 9,04 & 0,98 & 1,94 & 50 & 36 & 16 & - & 1,69 \\
\hline
\end{tabular}

P1, P2, P3, P4, P6: sitios de muestreo 1, 2, 3, 4, 6. Los demás sitios con datos por debajo del límite de detección (<LD).

Los sedimentos y la materia orgánica pueden retener metales por diferentes mecanismos, lo que disminuye la concentración en la columna de agua, pero los vínculos son débiles, un cambio de $\mathrm{pH}$, temperatura o intercambio catiónico o aniónico, presencia de carbonatos, entre otros puede liberarlos. Así, con esta inestabilidad, el riesgo que aumenten su concentración y biodisponibilidad en la CGSM es amplio, debido a las diversas fluctuaciones del ecosistema. De otra forma, $\mathrm{Cd}$ y $\mathrm{Pb}$ son absorbidos por las raíces de las plantas y transportados hacia sus tejidos por algunas especies bioacumuladoras que, cuando mueren, facilitan su sedimentación, pero, por el contrario, si son consumidas, el metal ingresa en la cadena trófica (Beltrame et al. 2009).

Siendo $\mathrm{Cd}$ y $\mathrm{Pb}$, metales calcófilos, tienen baja afinidad por $\mathrm{O}_{2}$ y se encuentran, principalmente, en forma de sulfuros insolubles. Por otra parte, un cambio en el porcentaje de salinidad o en oxígeno lo libera de los sedimentos hacia el agua (Koretsky et al. 2006). El Cd, se adhiere a la superficie de las partículas y así, fácilmente, es intercambiado y forma complejos con $\mathrm{Cl}$, donde puede quedar libre cuando la salinidad es alta. Al respecto, Lanjwani et al. (2020) encontraron correlación directa entre sales solubles (sodio, amonio, cloruros, nitratos y sulfatos), conductividad eléctrica y metales en sistemas estuarinos. Cuando el agua se evapora, las sales precipitan en forma de cristales y las costras protegen los residuos de la oxidación, evitando dejar libre los metales. Lo anterior es ratificado en la figura 2 , donde de muestra la tendencia de los elementos mencionados.

Además, Abouhend \& El-Moselhy (2015), concluyeron que la CE y el potencial redox tienen un efecto significativo, tanto en la adsorción como en la acumulación de metales en sedimentos; a un pH alto es más alta la concentración de Cd en los sedimentos, debido a la precipitación de $\mathrm{Cd}$ disuelto y la presencia $\mathrm{Pb}$, que fue más alta en los sedimentos, pero no disponible para los organismos acuáticos. Las fluctuaciones del sistema pueden inducir su liberación.

Con relación a las concentraciones de $\mathrm{Cr}$ y Ni, se observaron variaciones entre estaciones de muestreo, obteniéndose la máxima concentración en la estación Ciénaga de Machete, situación que coincide con INVEMAR (2016a), que indicó que Ni presentó valo-

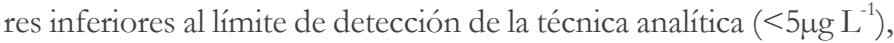
en la época lluviosa de 2013 y seca de 2014, siendo la concentración más alta hacia la cercanía del río Magdalena $\left(4,15 \mu \mathrm{g} \mathrm{L}^{-1}\right)$.
A partir del análisis, se evidenció concentración baja de oxígeno, aumento de sólidos, nutrientes disueltos y salinidad, variables que deterioran la calidad del agua de la CGSM. La contaminación por CT y CF hacen que no sea apta para contacto primario y podría afectar la supervivencia de la flora y fauna. La concentración de metales pesados fue baja, de acuerdo con los valores de referencia del Decreto 1076 de 2015 (Presidencia de la República de Colombia, 2015), pero su presencia en la columna de agua puede ser riesgoso para las poblaciones y el ambiente, puesto que las condiciones de fluctuación del complejo lagunar es alta y las concentraciones cambian, por lo cual, se sugiere realizar estudios más específicos en un periodo de tiempo mayor. Ante esta situación y el análisis ambiental es necesario realizar un monitoreo continuo y un plan de manejo del ecosistema.

Conflicto de intereses: El manuscrito fue preparado y revisado con la participación de todos los autores, quienes declaramos que no existe ningún conflicto de intereses que ponga en riesgo la validez de los resultados presentados.

\section{REFERENCIAS}

1. ABOUHEND, A.S.; EL-MOSELHY, K.M. 2015. Spatial and seasonal variations of heavy metals in water and sediments at the northern Red Sea coast. American Journal of Water Resources. 3(3):73-85.

https://doi.org/10.12691/ajwr-3-3-2

2. AMERICAN PUBLIC HEALTH ASSOCIATION, APHA; AMERICAN WATER WORKS ASSOCIATION, AWWA; WATER ENVIRONMENT. 2012. Standard methods for the examination of water and wastewater. Ed. APHAAWWA-WEF (Washington DC). 1496p.

3. BELTRAME, M.O.; DE MARCO, S.G.; MARCOVECCHIO, J.E. 2009. Dissolved and particulate heavy metals distribution in coastal lagoons. A case study from Mar Chiquita Lagoon, Argentina. Estuarine, Coastal and Shelf Science. 85(1):45-56.

https://doi.org/10.1016/j.ecss.2009.04.027 
4. CAETANO, M.; FONSECA, N.; VALE, R. 2007. Mobility of $\mathrm{Pb}$ in salt marshes recorded by total content and stable isotopic signature. Science of the Total Environment. 380(1-3):84-92.

https://doi.org/10.1016/j.scitotenv.2006.11.026

5. CAMPOS, C. 1990. La contaminación por metales pesados en la Ciénaga Grande de Santa Marta, Caribe colombiano. Caldasia. 16(77):231-244.

6. CAPORALE, A.G.; VIOLANTE, A. 2016. Chemical processes affecting the mobility of heavy metals and metalloids in soil environments. Current Pollution Reports. 2(1):15-27. https://doi.org/10.1007/s40726-015-0024-y

7. CUIZANO, N.A.; REYES, U.F.; DOMÍNGUEZ, S.; LLANOS, B.P.; NAVARRO, A.E. 2010. Relevancia del pH en la adsorción de iones metálicos mediante algas pardas. Revista de la Sociedad Química del Perú. 76(2):123-130.

8. FERNÁNDEZ, M.D.; BOTTTÉ, S.; HOFFMEYER, M.; MARCOVECCHIO, J. 2009. Spatial and temporal distribution of cadmium and copper in water and zooplankton in the Bahía Blanca estuary, Argentina. Estuarine. Coastal and Shelf Science. 85:57-66.

https://doi.org/10.1016/j.ecss.2009.03.019

9. HERNÁNDEZ-AYÓN, M.; ZIRINO, A.; MARINONE, S.G.; CANINO-HERRERA, R.; GALINDO-BECT, M.S. 2003. Relación pH-densidad en el agua de mar. Ciencias Marinas. 29(4):497-508.

10. INFOSTAT. 2015. Disponible desde Internet en: https://infostat.uptodown.com/windows/descargar

11. INSTITUTO DE ESTUDIOS PARA EL DESARROLLO Y LA PAZ, INDEPAZ. 2012. VIII Informe Sobre Grupos Narco paramilitares. Bogotá. Disponible desde internet en: http: / / www.indepaz.org.co/wp-content / uploads/2013/08/Informe-VIII-Indepaz-final.pdf. (con acceso el 20/03/2019).

12. INSTITUTO DE INVESTIGACIONES MARINAS, INVEMAR. 2016a. Monitoreo de las condiciones ambientales y los cambios estructurales y funcionales de las comunidades vegetales y de los recursos pesqueros durante la rehabilitación de la Ciénaga Grande de Santa Marta. Informe Técnico Final 2015, Volumen 14. Santa Marta. 181p.

13. INSTITUTO DE INVESTIGACIONES MARINAS, INVEMAR. 2016b. Concepto técnico sobre la mortandad de peces en la Ciénaga Grande de Santa Marta (sector Boca De La Barra), Magdalena, ocurrida en septiembre de 2016. CPT-CAM-033-16. 11p.
14. JARAMILLO-LONDOÑO, J.C.; AGUIRRE-RAMÍREZ, N.J. 2012. Cambios espacio-temporales del plancton en la ciénaga de Aayapel (Córdoba-Colombia), durante la época de menor nivel del agua. Caldasia. 34(1):213-226.

15. KIRWAN, M.L.; GUNTENSPERGEN, G.R.; MORRIS, J.T. 2009. Latitudinal trends in Spartina alterniflora productivity and the response of coastal marshes to global change. Global Change Biology. 15:1982-1989. https://doi.org/10.1111/j.1365-2486.2008.01834.x

16. KORETSKY, C.M.; HAAS, J.R.; MILLER, D.; NDENGA, N.T. 2006. Seasonal variations in pore water and sediment geochemistry of littoral lake sediments (Asylum Lake, MI, USA). Geochemical Transactions. 7(1):1-26. https://doi.org/10.1186/1467-4866-7-11

17. LANGSTON, W.J.; POPE, N.D.; JONAS, P.; NIKITIC, C.; FIELD, M.; DOWELL, B.; SHILLABEER, N.; SWARBRICK, R.; BROWN, A.R. 2010. Contaminants in fine sediments and their consequences for biota of the Severn Estuary. Marine pollution bulletin. 61:68-82. https://doi.org/10.1016/j.marpolbul.2009.12.014

18. LANJWANI, M.F.; KHUHAWAR, M.Y.; JAHANGIR KHUHAWAR, T.M.; LANJWANI, A.H.; JAGIRANI, M.S.; KORI, A.H.; RIND, I.K.; HUSSAIN, A.; MUHAMMAD, J. 2020. Risk assessment of heavy metals and salts for human and irrigation consumption of groundwater in Qambar city: a case study. Geology, Ecology, and Landscapes. 4(1):23-39. https://doi.org/10.1080/24749508.2019.1571670

19. MARCOVECCHIO J.; BOTTE, S.; FERNANDEZ, M.; DELUCCHI, F. 2010. Geochemical Control of Heavy Metal Concentrations and Distribution within Bahia Blanca Est. (Argentina). Aquatic Geochemistry. 16(2):251-266. https://doi.org/10.1007/s10498-009-9076-1

20. MARCOVECCHIO, J.; FREIJE, R. 2013. Procesos químicos en Estuarios. Universidad Tecnológica Nacional, México. $394 \mathrm{p}$.

21. MARTIINEZ, A.S.; HINOJOSA, S.; ROZO, O.S. 2009. Proceso y avance hacia la sostenibilidad ambiental: la reserva de biosfera Seaflower, en el caribe colombiano. Cuadernos del Caribe. 7(13):7-23.

22. MINISTERIO DE AMBIENTE Y DESARROLLO SOSTENIBLE, MINAMBIENTE. 2018. Resolución 0618. República de Colombia. Disponible desde Internet en: http://www.minambiente.gov.co/images/normativa/app/ resoluciones/ed-res\%20618\%20de\%202018.pdf (con acceso el 07/04/2019). 
23. PÉREZ ORTEGA, D.J.; SEGOVIA ORTEGA, J.A.; CABRERA MONCAYO, P.C.; DELGADO VARGAS, I.A.; MARTINS POMPÊO, M.L. 2018. Uso del suelo y su influencia en la presión y degradación de los recursos hídricos en cuencas hidrográficas. Rev. Investigación Agraria y Ambiental. 9(1):41-57. https://doi.org/10.22490/21456453.2089

24. PRADANA, J.A.; GALLEGO, A.; GARCÍA, J.; BRAVO, J.; GARCÍA, M.; PANIAGUA, G. 2019. Criterios de calidad y gestión del agua potable. Ed. UNED (Madrid - España). $467 \mathrm{p}$.

25. PRESIDENCIA DE LA REPÚBLICA DE COLOMBIA. 2015. Decreto 1076 de 2015. Disponible desde Internet en: https://www.habitatbogota.gov.co/decreto-1076-2015 (con acceso 05/08/2020).

26. R CORE TEAM. 2018. A Language and Environment for Statistical Computing. R Foundation for Statistical Computing, Vienna.

https:/ /www.R-project.org

27. REYES, Y.C.; VERGARA, I.; TORRES, O.E.; DIAZ, M.; GONZALEZ E.E. 2016. Contaminación por metales pesados: implicaciones en salud, ambiente y seguridad alimentaria. Ingeniería, Investigación y Desarrollo. 16(2):6677.

28. RODRÍGUEZ-RODRÍGUEZ, J.A.; PINEDA, J.; TRUJILLO, L.; RUEDA, M.; IBARRA-GUTIÉRREZ, K. 2016. Cié- naga Grande de Santa Marta: The Largest Lagoon-Delta Ecosystem in the Colombian Caribbean. In: Milton, G.R., Prentice, R.C.; Finlayson, C.M. (eds.). The Wetland Book: II: Distribution, Description and Conservation. p.1-16. Springer, Dordrecht Switzerland. https://doi.org/10.1007/978-94-007-6173-5_126-1

29. SARMIENTO, J.P. 2016. Justicia transicional sin transición. El caso de la masacre de Nueva Venecia. Co-herencia. 13(24):181-211. http://dx.doi.org/10.17230/co-herencia.13.24.7

30. SPETTER, C.V.; POPOVICH, C.; ARIAS, A.; ASTEASUAIN, R.; FREIJE, R.; MARCOVECCHIO, J.E. 2013. Role of nutrients in phytoplankton development during a winter diatom bloom in a eutrophic South American estuary (Bahía Blanca, Argentina). J. Coastal Research. 31(1):76-87. https://doi.org/10.2112/JCOASTRES-D-12-00251.1

31. VIVAS-AGUAS, L.J.; ESPINOZA, L.F; PARRA-HENRÍQUEZ, L.G. 2013. Identificación de fuentes terrestres de contaminación y cálculo de las cargas de contaminantes en el área de influencia de la Ciénaga Grande de Santa Marta, Caribe Colombiano. Boletín de Investigaciones Marinas y Costeras-INVEMAR. 42(1):7-30.

https://doi.org/10.25268/bimc.invemar.2013.42.1.57

32. YÁÑEZ-ARANCIBIA, A.; DAY, J.W.; TWILLEY, R.R.; DAY, R.H. 2014. Manglares: ecosistema centinela frente al cambio climático, Golfo de México. Madera y Bosques. 20:3975. 